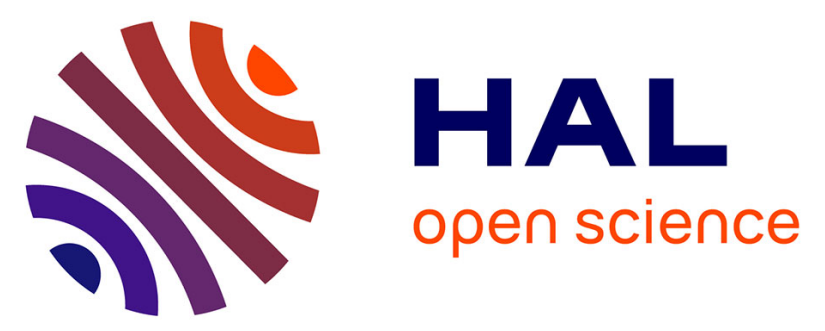

\title{
Wave Finite Element based Strategies for Computing the Acoustic Radiation of Stiffened or Non-Stiffened Rectangular Plates subject to Arbitrary Boundary Conditions
}

\author{
Jean-Mathieu Mencik, Marie-Laure Gobert
}

\section{To cite this version:}

Jean-Mathieu Mencik, Marie-Laure Gobert. Wave Finite Element based Strategies for Computing the Acoustic Radiation of Stiffened or Non-Stiffened Rectangular Plates subject to Arbitrary Boundary Conditions. The Eleventh International Conference on Computational Structures Technology, Sep 2012, Dubrovnik, Croatia. Civil-Comp Press, Stirlingshire, UK, Paper 218, 2012, 10.4203/ccp.99.218 . hal-00816783

\section{HAL Id: hal-00816783 \\ https://hal.science/hal-00816783}

Submitted on 23 Apr 2013

HAL is a multi-disciplinary open access archive for the deposit and dissemination of scientific research documents, whether they are published or not. The documents may come from teaching and research institutions in France or abroad, or from public or private research centers.
L'archive ouverte pluridisciplinaire HAL, est destinée au dépôt et à la diffusion de documents scientifiques de niveau recherche, publiés ou non, émanant des établissements d'enseignement et de recherche français ou étrangers, des laboratoires publics ou privés. 


\title{
Wave finite element based strategies for computing the acoustic radiation of stiffened or non-stiffened rectangular plates subject to arbitrary boundary conditions
}

\author{
J.-M. Mencik and M.-L. Gobert \\ ENI Val de Loire, Université François Rabelais de Tours, LMR, Rue de la Chocolaterie, BP 3410, \\ F-41034 Blois Cedex, France \\ Email: jean-mathieu.mencik@univ-tours.fr
}

\begin{abstract}
The wave finite element method (WFE) is investigated for the computation of the acoustic radiation of stiffened or non-stiffened rectangular plates under arbitrary boundary conditions. The method aims at computing the forced response of periodic waveguides (e.g. rectangular plates that are homogeneous or that contain a periodic distribution of stiffeners) using numerical wave modes. A WFE-based strategy is proposed which uses the method of elementary radiators for expressing the radiation efficiencies of stiffened or non-stiffened baffled rectangular plates immersed in a light acoustic fluid. In addition, a model reduction strategy consisting in using reduced wave bases for computing these radiation efficiencies with small CPU times is proposed. Numerical experiments highlight the relevance of the strategies.
\end{abstract}

Keywords: wave finite elements, model reduction, mid-frequencies, acoustic radiation.

\section{Introduction}

The wave finite element (WFE) method is investigated for the computation of the acoustic radiation of stiffened or non-stiffened rectangular plates under arbitrary boundary conditions. The method aims at numerically providing the waves traveling in positive and negative directions along periodic waveguides, i.e. elastic structures that are assumed to be modeled by means of identical substructures connected along a main axis (namely, the direction of propagation). In fact, stiffened or non-stiffened rectangular plates that are meshed periodically along their length belong to that class of waveguides. The WFE method uses the finite element (FE) model of a typical substructure to compute numerical wave modes. These are to be understood as particular shapes of the displacement and force fields over the system cross-section, "traveling" with different velocities along the waveguide. The WFE method enables the 
propagating, evanescent and complex wave modes to be captured over the low and mid-frequency range. Using these wave modes as representation bases constitutes an efficient means for computing the forced response of waveguides under arbitrary boundary conditions [1].

In this work, a WFE-based strategy that uses the aforementioned wave modes for expressing the radiation efficiencies of stiffened or non-stiffened rectangular plates is proposed. Such plates are assumed to be surrounded by an infinite rigid baffle while radiating in a light acoustic fluid. The radiation efficiencies are computed using the method of elementary radiators [2], which requires us to discretize the plates into small surfaces while expressing the normal velocities of these elementary surfaces in terms of wave modes. In comparison to the classic FE method, the feature of the proposed WFE formulation is that it exhibits matrices which do not depend on the waveguide boundary conditions (i.e. over the limiting ends where reflection of waves occur), meaning that it can be reused with less computational time to address changes of those boundary conditions.

In addition, a model order reduction (MOR) strategy consisting in using reduced wave bases for computing these radiation efficiencies is proposed. The motivation behind this work is to reduce significantly the computational times compared to the case when the full wave bases are used in the WFE matrix formulations (it is worth noting that, even in the WFE framework, the CPU times required to compute the radiation efficiency of a plate at many discrete frequencies can be substantial). For any waveguide, a norm-wise error analysis is proposed for efficiently reducing the size of the wave basis involved in the description of the dynamic behavior. The proposed MOR strategy has been fully investigated in a previous work [3]. The key idea behind the strategy is to invoke a finite number of forward / backward passings of waves along the waveguide for expressing the wave amplitudes. This yields the error induced for expressing the displacements and forces of the waveguide to be bounded by means of matrix norms that are not necessarily decreasing functions of the number of retained wave modes. The resulting error bound is found to be sensitive (that is, it increases) when the wave basis tend to be oversized. It is shown that the minimum of this error bound provides the exact number of wave modes to be retained for the computation of the forced response of the waveguide.

The rest of the paper is organized as follows. In Section 2, the WFE framework is recalled. The WFE-based strategy for computing the radiation efficiencies of stiffened or non-stiffened baffled rectangular plates radiating in a light acoustic fluid is proposed in Section 3. The WFE-based MOR strategy which yields the radiation efficiencies of these plates to be described in terms of reduced wave bases of small sizes is proposed in Section 4. Numerical experiments are brought in Section 5; the accuracy and relevance of the proposed strategies are highlighted compared to the classic FE method as well as analytical theories. 


\section{WFE method}

\subsection{Theory}

The WFE method aims at numerically describing the waves traveling along periodic structures [4]. Such structures are called periodic in the sense that their FE models is described by means of identical substructures that are connected along a main axis $x$ (namely, the direction of wave propagation). Rectangular plates which are meshed "periodically" along their length ( $x$-direction) belong to that class of structures. In the present study, these plates are supposed to be elastic, dissipative (considering a loss factor $\eta$ ) and subjected to harmonic disturbance under frequency $\omega / 2 \pi$ ( $\omega$ being the pulsation). A rectangular plate with a periodic FE mesh is shown in Figure 1. The related substructures have the same length $d$ while their left and right boundaries (i.e. the edges coincident with the $y$-direction) contain the same number of degrees of freedom (DOFs), namely $n$ (cf. Figure 1).
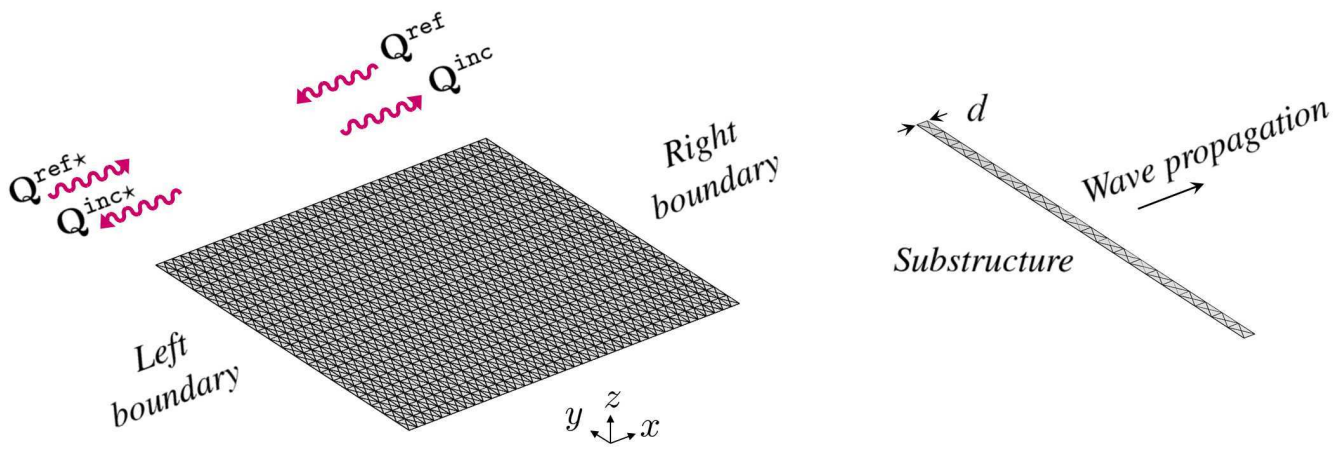

Figure 1: Illustration of waves traveling along a rectangular plate ( $x$-direction); FE model of a representative substructure.

Within the WFE framework, the waves traveling along the $x$-direction of the structure are to be described. The computation of the so-called wave modes follows from Bloch's theorem, considering the FE model of a representative substructure of the whole system (see Figure 1). Once the dynamic stiffness matrix of the substructure is known (e.g. using a commercial FE software), a state vector representation [5] that links the kinematic / mechanical fields - i.e. the translational and rotational displacements, as well as the forces and moments - between the left (or right) boundaries of two adjacent substructures $k$ and $k-1$ can be expressed as [1]

$$
\mathbf{u}^{(k)}=\mathbf{S} \mathbf{u}^{(k-1)},
$$

where $\mathbf{S}$ is a $2 n \times 2 n$ symplectic matrix. Also, $\mathbf{u}$ is to be understood as a $2 n \times 1$ state vector expressed as

$$
\mathbf{u}=\left[\begin{array}{c}
\mathbf{q} \\
\pm \mathbf{F}
\end{array}\right]
$$


where $\mathbf{q}$ and $\mathbf{F}$ are $n \times 1$ vectors which denote, respectively, the translational / rotational displacements and the forces / moments over the substructure boundaries. The sign ahead $\mathbf{F}$ results from the convention made for expressing the forces on the left or right boundaries of the substructures: in the present case, the conventions $-\mathbf{F}$ (resp. F) will be used to denote the left (resp. right) boundary of each substructure.

What states Bloch's theorem is that the eigenvalues of $\mathbf{S}$ - namely $\left\{\mu_{j}\right\}_{j}$ - can be expressed as $\left\{\mathrm{e}^{-i \beta_{j} d}\right\}_{j}$, where $\left\{\beta_{j}\right\}_{j}$ have the meaning of wavenumbers. Also, the terms $\left\{\boldsymbol{\Phi}_{j}\right\}_{j}$ are the eigenvectors of $\mathbf{S}-$ also known as wave shapes -, which relate the spatial distribution of the kinematic and mechanical fields over the width of the plate (i.e. along the $y$-direction). The wave modes usually refer to the set of parameters $\left\{\left(\mu_{j}, \boldsymbol{\Phi}_{j}\right)\right\}_{j}$, or simply the wave shapes $\left\{\boldsymbol{\Phi}_{j}\right\}_{j}$. Considering that the matrix $\mathbf{S}$ is symplectic (see above) yields $\left\{\left(\mu_{j}, \boldsymbol{\Phi}_{j}\right)\right\}_{j}$ to be split into $n$ incident and $n$ reflected wave modes, i.e. $n$ waves traveling towards and $n$ waves traveling away from the right (or left) boundary of the waveguide. These incident and reflected wave modes are denoted as $\left\{\left(\mu_{j}^{\text {inc }}, \boldsymbol{\Phi}_{j}^{\text {inc }}\right)\right\}_{j}$ and $\left\{\left(\mu_{j}^{\text {ref }}, \boldsymbol{\Phi}_{j}^{\text {ref }}\right)\right\}_{j}$; they are usually defined so that $\left|\mu_{j}^{\text {inc }}\right|<1$ and $\left|\mu_{j}^{\text {ref }}\right|>1 \forall j$ (such a consideration follows from the fact that $\mathbf{S}$ is a symplectic matrix - i.e. its eigenvalues come in pairs as $(\mu, 1 / \mu)$ - while it is assumed that the structure is damped).

Convention. The notations $\left\{\boldsymbol{\Phi}_{j}^{\text {inc }}\right\}_{j}$ and $\left\{\boldsymbol{\Phi}_{j}^{\text {ref }}\right\}_{j}$ will be used throughout the paper to denote the waves modes traveling towards and away from the right boundary of the waveguide. In contrast, the notations $\left\{\boldsymbol{\Phi}_{j}^{\text {inc*}}\right\}_{j}$ and $\left\{\boldsymbol{\Phi}_{j}^{\text {ref }}\right\}_{j}$ will be used to denote the incident / reflected wave modes with regard to the left boundary of the waveguide (see Figure 1). Those wave modes are simply linked as $\Phi_{j}^{\text {inc }}=\Phi_{j}^{\text {ref }}$ and $\Phi_{j}^{\text {ref }}=\boldsymbol{\Phi}_{j}^{\text {inc }}$ $\forall j$. This convention is introduced here as a means to simplify the subsequent developments made in the paper.

Finally, Bloch's theorem states that the vectors of displacements q and forces / moments $\pm \mathbf{F}$, over any substructure boundary $k$ (i.e. either a coupling interface between two consecutive substructures $k-1$ and $k$, or a limiting edge of the waveguide), can be expanded in terms of wave modes as [1]

$$
\mathbf{q}^{(k)}=\boldsymbol{\Phi}_{\mathrm{q}}^{\mathrm{inc}} \mathrm{Q}^{\mathrm{inc}(k)}+\boldsymbol{\Phi}_{\mathrm{q}}^{\mathrm{ref}} \mathbf{Q}^{\mathrm{ref}(k)} \quad, \quad \pm \mathbf{F}^{(k)}=\boldsymbol{\Phi}_{\mathrm{F}}^{\mathrm{inc}} \mathbf{Q}^{\mathrm{inc}(k)}+\boldsymbol{\Phi}_{\mathrm{F}}^{\mathrm{ref}} \mathrm{Q}^{\mathrm{ref}(k)},
$$

where $\Phi_{\mathrm{q}}^{\text {inc }}, \Phi_{\mathrm{q}}^{\text {ref }}, \boldsymbol{\Phi}_{\mathrm{F}}^{\text {inc }}$ and $\boldsymbol{\Phi}_{\mathrm{F}}^{\text {ref }}$ are square $n \times n$ matrices constituted from the displacement and force / moment components of the incident and reflected wave shapes; also, $\mathrm{Q}^{\text {inc(k) }}$ and $\mathrm{Q}^{\mathrm{ref}(k)}$ are $n \times 1$ vectors of wave amplitudes whose variation along the waveguide is governed as [1]

$$
\mathrm{Q}^{\mathrm{inc}(k)}=\boldsymbol{\mu} \mathrm{Q}^{\mathrm{inc}(k-1)} \quad, \quad \mathrm{Q}^{\mathrm{ref}(k)}=\boldsymbol{\mu}^{-1} \mathrm{Q}^{\mathrm{ref}(k-1)},
$$

where $\boldsymbol{\mu}$ is a $n \times n$ matrix defined as $\boldsymbol{\mu}=\operatorname{diag}\left\{\mu_{j}^{\text {inc }}\right\}_{j}$, such that $\|\boldsymbol{\mu}\|_{2}<1\left(\|\cdot\|_{2}\right.$ being the $2-$ norm $)^{1}$. Considering the aforementioned convention regarding incident

\footnotetext{
${ }^{1}$ The fact that $\|\boldsymbol{\mu}\|_{2}<1$ is readily proved since $\left|\mu_{j}^{\text {inc }}\right|<1 \forall j$ (see above).
} 
/ reflected waves, yields the wave mode expansion (3) to be rewritten as

$$
\mathbf{q}^{(k)}=\boldsymbol{\Phi}_{\mathrm{q}}^{\mathrm{ref} \star} \mathbf{Q}^{\mathrm{ref} \star(k)}+\boldsymbol{\Phi}_{\mathrm{q}}^{\mathrm{ref}} \mathbf{Q}^{\mathrm{ref}(k)}, \quad \pm \mathbf{F}^{(k)}=\boldsymbol{\Phi}_{\mathrm{F}}^{\mathrm{ref} \star} \mathbf{Q}^{\mathrm{ref} \star(k)}+\boldsymbol{\Phi}_{\mathrm{F}}^{\mathrm{ref}} \mathbf{Q}^{\mathrm{ref}(k)},
$$

where $\Phi^{\text {ref } \star}$ and $\mathrm{Q}^{\text {ref } \star(k)}$ refer to the wave modes which are reflected by the left boundary of the waveguide (see above), with the convention $\Phi^{\text {ref } \star}=\Phi^{\text {inc }}$ and $\mathbf{Q}^{\text {ref } \star(k)}=$ $\mathrm{Q}^{\text {inc*(k)}}$.

\subsection{Forced response computation: application to plates under ar- bitrary boundary conditions excited by a point force}

The forced response computation of rectangular plates excited by a point force and whose edges are subjected to arbitrary boundary conditions is investigated using the WFE method. As an example, simply supported rectangular plates (whose related FE model is depicted in Figure 2) represent a particular kind of structures addressed within the present study. As discussed in the previous section, the FE models of these structures are assumed to be periodic in the sense they can be described by means of identical substructures (say, whose total number is $N$ ) along a specific $x$-direction (see Figure 2). It is worth noting that, in the present case, the substructures are supposed to exhibit the same boundary conditions as the whole structure on the edges parallel to the $x$-direction (say along the length $d$ ).
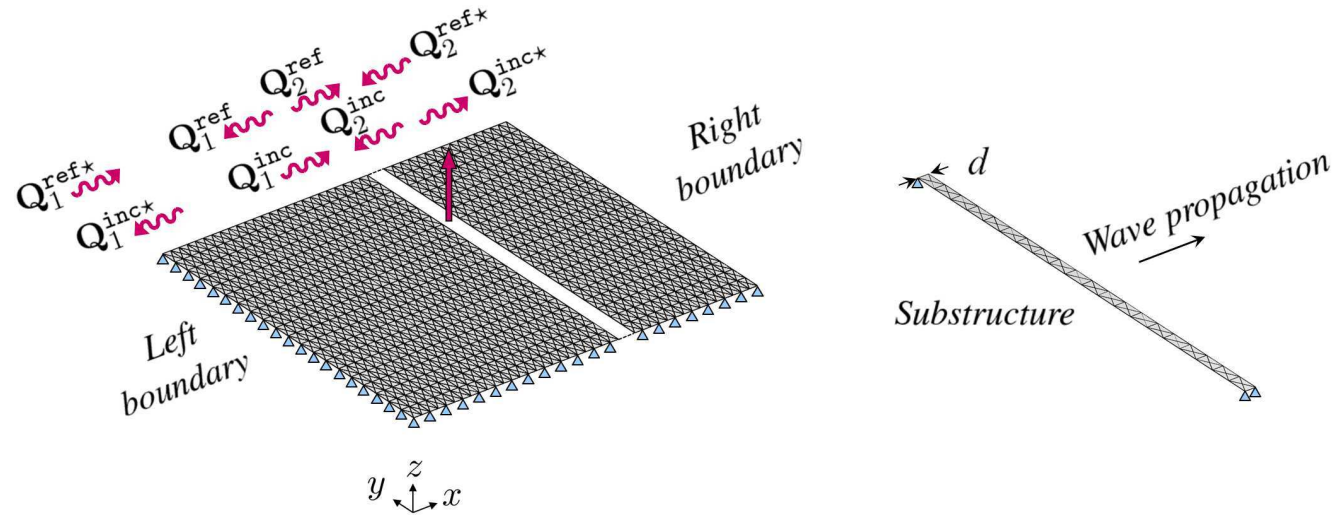

Figure 2: Wave-based description of a simply supported rectangular plate excited by a point force and FE model of a representative substructure.

The WFE method aims at describing the kinematic and mechanical fields of such a rectangular plate, excited by a point force, in terms of wave modes (see above). The procedure consists in partitioning the whole structure into two connected waveguides 1 and 2 - i.e. two subplates respectively defined from the left and right boundaries of the whole structure, until the abscissa $x$ where the point force applies - which are composed from $N_{1}$ and $N_{2}$ substructures, respectively. Such a partitioning is highlighted in Figure 2. 
Let us denote as $\mathbf{q}_{i}^{\left(k_{i}\right)}$ and $\mathbf{F}_{i}^{\left(k_{i}\right)}$ the vectors of displacements and forces / moments (respectively) expressed over the substructure boundary $k_{i}$ of any waveguide $i$ ( $i=$ 1,2 ). The substructure boundaries for waveguide 1 (resp. waveguide 2) are numbered from 1 to $N_{i}+1$ from the left end (resp. the right end) until the location of the point force. The boundary conditions of waveguides 1 and 2 - i.e. over the left end of waveguide 1 and the right end of waveguide 2 - are supposed to be expressed under the following general form:

$$
\left(\mathcal{L}_{\mathrm{q}}\right)_{1} \mathbf{q}_{1}^{(1)}=\mathbf{0} \quad, \quad\left(\mathcal{L}_{\mathrm{F}}\right)_{1} \mathbf{F}_{1}^{(1)}=\mathbf{0} \quad, \quad\left(\mathcal{L}_{\mathrm{q}}\right)_{2} \mathbf{q}_{2}^{(1)}=\mathbf{0} \quad, \quad\left(\mathcal{L}_{\mathrm{F}}\right)_{2} \mathbf{F}_{2}^{(1)}=\mathbf{0},
$$

where $\left(\mathcal{L}_{\mathrm{q}}\right)_{i}$ and $\left(\mathcal{L}_{\mathrm{F}}\right)_{i}$ are two incidence matrices used to denote the particular displacement / force components that are equal to zero ${ }^{2}$. Also, it is implicitly supposed that the other boundary conditions of the waveguides - i.e. over their edges parallel to the $x$-direction - are expressed by means of Eq. (6). On the other hand, the coupling conditions between the two waveguides are expressed as

$$
\mathbf{q}_{1}^{\left(N_{1}+1\right)}=\mathbf{q}_{2}^{\left(N_{2}+1\right)} \quad, \quad \mathbf{F}_{1}^{\left(N_{1}+1\right)}=-\mathbf{F}_{2}^{\left(N_{2}+1\right)}+\mathbf{F}_{e x}
$$

where $\mathbf{F}_{e x}$ is a $n \times 1$ vector ( $n$ being the number of DOFs used for discretizing any substructure boundary) which denotes the forces applied on the coupling interface. In the present case where one single force occurs, only one component of $\mathbf{F}_{e x}$ is different from zero. In Eq. (7), $\mathbf{F}_{1}^{\left(N_{1}+1\right)}$ and $\mathbf{F}_{2}^{\left(N_{2}+1\right)}$ are to be understood as the vectors of internal forces respectively defined on the right boundary of waveguide 1 and the left boundary of waveguide 2 .

Within the WFE framework, a wave mode expansion of the form (5) is considered for each waveguide $i(i=1,2)$ as

$$
\begin{aligned}
& \mathbf{q}_{i}^{(k)}=\left(\Phi_{\mathrm{q}}^{\mathrm{ref} \star}\right)_{i} \mathbf{Q}_{i}^{\mathrm{ref} \star\left(k_{i}\right)}+\left(\boldsymbol{\Phi}_{\mathrm{q}}^{\mathrm{ref}}\right)_{i} \mathbf{Q}_{i}^{\mathrm{ref}\left(k_{i}\right)} \\
& \pm \mathbf{F}^{(k)}=\left(\boldsymbol{\Phi}_{\mathrm{F}}^{\mathrm{ref} \star}\right)_{i} \mathbf{Q}_{i}^{\mathrm{ref} \star\left(k_{i}\right)}+\left(\boldsymbol{\Phi}_{\mathrm{F}}^{\mathrm{ref}}\right)_{i} \mathbf{Q}_{i}^{\mathrm{ref}\left(k_{i}\right)} \quad k_{i}=1, \ldots, N_{i}+1 \quad i=1,2,
\end{aligned}
$$

where $\mathbf{Q}_{i}^{\text {ref } \star\left(k_{i}\right)}$ and $\mathbf{Q}_{i}^{\text {ref }\left(k_{i}\right)}$ are the $n \times 1$ vectors of wave amplitudes for waveguide $i$, defined at the substructure boundary $k_{i}$ (i.e. either a coupling interface between two substructures, or one limiting edge of the waveguide), while $\left(\boldsymbol{\Phi}_{\mathrm{q}}^{\text {ref } \star}\right)_{2}=\left(\Phi_{\mathrm{q}}^{\text {ref }}\right)_{1}$ and $\left(\boldsymbol{\Phi}_{\mathrm{F}}^{\text {ref }}\right)_{2}=\left(\boldsymbol{\Phi}_{\mathrm{F}}^{\text {ref }}\right)_{1}$. Notice that wave modes traveling along the two waveguides are similar. The only change concerns the vectors of wave amplitudes which, due to the discontinuity of the internal force field across the interface where excitation source occurs, are to be considered different between the two waveguides. Considering the aforementioned wave mode expansion enables the boundary conditions and coupling conditions of these two waveguides to be expressed in wave-based form. For this task, the following simplified notations are introduced:

$$
\mathbf{Q}_{i}^{\mathrm{ref}\left(N_{i}+1\right)}=\mathbf{Q}_{i}^{\mathrm{ref}} \quad, \quad \mathbf{Q}_{i}^{\mathrm{ref} \star(1)}=\mathbf{Q}_{i}^{\mathrm{ref} \star} \quad i=1,2 .
$$

\footnotetext{
${ }^{2}$ For instance, considering simply supported boundary conditions yields both transverse displacements and bending moments to be zero; in this case, one has $\left(\mathcal{L}_{\mathrm{q}}\right)_{1}=\left(\mathcal{L}_{\mathrm{q}}\right)_{2}$ and $\left.\left(\mathcal{L}_{\mathrm{F}}\right)_{1}=\left(\mathcal{L}_{\mathrm{F}}\right)_{2}\right)$.
} 
Expressing Eqs. (6) and (7) in wave-based form while using the simplified notations provided by Eq. (9) and the governing equations (4) results in

$$
\begin{aligned}
& \left(\mathcal{L}_{\mathrm{q}}\right)_{i}\left[\left(\boldsymbol{\Phi}_{\mathrm{q}}^{\mathrm{ref} \star}\right)_{i} \mathbf{Q}_{i}^{\text {ref } \star}+\left(\boldsymbol{\Phi}_{\mathrm{q}}^{\text {ref }}\right)_{i} \boldsymbol{\mu}^{N_{i}} \mathbf{Q}_{i}^{\text {ref }}\right]=\mathbf{0}, \\
& \left(\mathcal{L}_{\mathrm{F}}\right)_{i}\left[\left(\boldsymbol{\Phi}_{\mathrm{F}}^{\text {ref } \star}\right)_{i} \mathbf{Q}_{i}^{\text {ref } \star}+\left(\boldsymbol{\Phi}_{\mathrm{F}}^{\text {ref }}\right)_{i} \boldsymbol{\mu}^{N_{i}} \mathbf{Q}_{i}^{\text {ref }}\right]=\mathbf{0} \quad i=1,2 .
\end{aligned}
$$

and

$$
\begin{aligned}
& {\left[\left(\boldsymbol{\Phi}_{\mathrm{q}}^{\text {ref } \star}\right)_{1} \boldsymbol{\mu}^{N_{1}} \mathbf{Q}_{1}^{\text {ref } \star}+\left(\boldsymbol{\Phi}_{\mathrm{q}}^{\text {ref }}\right)_{1} \mathbf{Q}_{1}^{\text {ref }}\right]=\left[\left(\boldsymbol{\Phi}_{\mathrm{q}}^{\text {ref } \star}\right)_{2} \boldsymbol{\mu}^{N_{2}} \mathbf{Q}_{2}^{\text {ref } \star}+\left(\boldsymbol{\Phi}_{\mathrm{q}}^{\text {ref }}\right)_{2} \mathbf{Q}_{2}^{\text {ref }}\right],} \\
& {\left[\left(\boldsymbol{\Phi}_{\mathrm{F}}^{\text {ref } \star}\right)_{1} \boldsymbol{\mu}^{N_{1}} \mathbf{Q}_{1}^{\text {ref } \star}+\left(\boldsymbol{\Phi}_{\mathrm{F}}^{\text {ref }}\right)_{1} \mathbf{Q}_{1}^{\text {ref }}\right]=\left[\left(\boldsymbol{\Phi}_{\mathrm{F}}^{\text {ref } \star}\right)_{2} \boldsymbol{\mu}^{N_{1}} \mathbf{Q}_{2}^{\text {ref } \star}+\left(\boldsymbol{\Phi}_{\mathrm{F}}^{\text {ref }}\right)_{2} \mathbf{Q}_{2}^{\text {ref }}\right]+\mathbf{F}_{\text {ex }}}
\end{aligned}
$$

In matrix form, these relationships yield

$$
\left[\begin{array}{c}
\left(\mathcal{L}_{\mathrm{q}}\right)_{i}\left(\boldsymbol{\Phi}_{\mathrm{q}}^{\mathrm{ref} \star}\right)_{i} \\
\left(\mathcal{L}_{\mathrm{F}}\right)_{i}\left(\mathbf{\Phi}_{\mathrm{F}}^{\mathrm{ref} \star}\right)_{i}
\end{array}\right] \mathbf{Q}_{i}^{\mathrm{ref} \star}=-\left[\begin{array}{c}
\left(\mathcal{L}_{\mathrm{q}}\right)_{i}\left(\boldsymbol{\Phi}_{\mathrm{q}}^{\mathrm{ref}}\right)_{i} \\
\left(\mathcal{L}_{\mathrm{F}}\right)_{i}\left(\boldsymbol{\Phi}_{\mathrm{F}}^{\text {ref }}\right)_{i}
\end{array}\right] \boldsymbol{\mu}^{N_{i}} \mathbf{Q}_{i}^{\text {ref }} \quad i=1,2,
$$

and

$$
\left[\begin{array}{cc}
\left(\boldsymbol{\Phi}_{\mathrm{q}}^{\mathrm{ref}}\right)_{1} & -\left(\boldsymbol{\Phi}_{\mathrm{q}}^{\mathrm{ref}}\right)_{2} \\
-\left(\mathbf{\Phi}_{\mathrm{F}}^{\mathrm{ref}}\right)_{1} & \left(\boldsymbol{\Phi}_{\mathrm{F}}^{\mathrm{ref}}\right)_{2}
\end{array}\right]\left[\begin{array}{c}
\mathbf{Q}_{1}^{\mathrm{ref}} \\
\mathbf{Q}_{2}^{\text {ref }}
\end{array}\right]=-\left[\begin{array}{cc}
\left(\boldsymbol{\Phi}_{\mathrm{q}}^{\mathrm{ref} \star}\right)_{1} & -\left(\boldsymbol{\Phi}_{\mathrm{q}}^{\mathrm{ref} \star}\right)_{2} \\
-\left(\boldsymbol{\Phi}_{\mathrm{F}}^{\mathrm{ref} \star}\right)_{1} & \left(\boldsymbol{\Phi}_{\mathrm{F}}^{\mathrm{ref}}\right)_{2}
\end{array}\right]\left[\begin{array}{c}
\boldsymbol{\mu}^{N_{1}} \mathbf{Q}_{1}^{\mathrm{ref} \star} \\
\boldsymbol{\mu}^{N_{2}} \mathbf{Q}_{2}^{\text {ref } \star}
\end{array}\right]-\left[\begin{array}{c}
\mathbf{0} \\
\mathbf{F}_{e x}
\end{array}\right] .
$$

Invoking matrix inverses enables these equations to be expressed in the following compact forms:

$$
\mathbf{Q}_{i}^{\text {ref } \star}=\mathbb{C}_{i}^{\star} \boldsymbol{\mu}^{N_{i}} \mathbf{Q}_{i}^{\text {ref }} \quad i=1,2 \quad, \quad\left[\begin{array}{l}
\mathbf{Q}_{1}^{\text {ref }} \\
\mathbf{Q}_{2}^{\text {ref }}
\end{array}\right]=\mathbb{C}\left[\begin{array}{c}
\boldsymbol{\mu}^{N_{1}} \mathbf{Q}_{1}^{\text {ref } \star} \\
\boldsymbol{\mu}^{N_{2}} \mathbf{Q}_{2}^{\text {ref }}
\end{array}\right]+\mathbb{F} .
$$

where $\mathbb{C}_{i}^{\star}$ is a $n \times n$ matrix whose components denote the reflection coefficients of wave modes, at the left end of waveguide 1 (case when $i=1$ ) and the right end of waveguide 2 (case when $i=2$ ). This matrix is expressed as

$$
\mathbb{C}_{i}^{\star}=-\left[\begin{array}{c}
\left(\mathcal{L}_{\mathrm{q}}\right)_{i}\left(\boldsymbol{\Phi}_{\mathrm{q}}^{\mathrm{ref} \star}\right)_{i} \\
\left(\mathcal{L}_{\mathrm{F}}\right)_{i}\left(\boldsymbol{\Phi}_{\mathrm{F}}^{\mathrm{ref} \star}\right)_{i}
\end{array}\right]^{-1}\left[\begin{array}{c}
\left(\mathcal{L}_{\mathrm{q}}\right)_{i}\left(\boldsymbol{\Phi}_{\mathrm{q}}^{\mathrm{ref}}\right)_{i} \\
\left(\mathcal{L}_{\mathrm{F}}\right)_{i}\left(\boldsymbol{\Phi}_{\mathrm{F}}^{\mathrm{ref}}\right)_{i}
\end{array}\right] \quad i=1,2
$$

Also, in Eq. (14), $\mathbb{C}$ is a $2 n \times 2 n$ matrix expressed as

$$
\mathbb{C}=-\left[\begin{array}{cc}
\mathbf{I} & -\left(\boldsymbol{\Phi}_{\mathrm{q}}^{\mathrm{ref}}\right)_{1}^{-1}\left(\boldsymbol{\Phi}_{\mathrm{q}}^{\mathrm{ref}}\right)_{2} \\
-\left(\boldsymbol{\Phi}_{\mathrm{F}}^{\mathrm{ref}}\right)_{2}^{-1}\left(\boldsymbol{\Phi}_{\mathrm{F}}^{\mathrm{ref}}\right)_{1} & \mathbf{I}
\end{array}\right]^{-1}\left[\begin{array}{cc}
\left(\boldsymbol{\Phi}_{\mathrm{q}}^{\mathrm{ref}}\right)_{1}^{-1}\left(\boldsymbol{\Phi}_{\mathrm{q}}^{\mathrm{ref} \star}\right)_{1} & -\left(\boldsymbol{\Phi}_{\mathrm{q}}^{\mathrm{ref}}\right)_{1}^{-1}\left(\boldsymbol{\Phi}_{\mathrm{q}}^{\mathrm{ref} \star}\right)_{2} \\
-\left(\boldsymbol{\Phi}_{\mathrm{F}}^{\mathrm{ref}}\right)_{2}^{-1}\left(\boldsymbol{\Phi}_{\mathrm{F}}^{\mathrm{ref} \star}\right)_{1} & \left(\boldsymbol{\Phi}_{\mathrm{F}}^{\mathrm{ref}}\right)_{2}^{-1}\left(\boldsymbol{\Phi}_{\mathrm{F}}^{\mathrm{ref} \star}\right)_{2}
\end{array}\right] .
$$

The matrix $\mathbb{C}$ can be partitioned as

$$
\mathbb{C}=\left[\begin{array}{ll}
\mathbb{C}_{11} & \mathbb{C}_{12} \\
\mathbb{C}_{21} & \mathbb{C}_{22}
\end{array}\right]
$$

where $\mathbb{C}_{11}$ and $\mathbb{C}_{22}$ are $n \times n$ matrices whose components represent the reflection coefficients of wave modes at the coupling interface, while $\mathbb{C}_{12}$ and $\mathbb{C}_{21}$ are $n \times n$ matrices 
whose components represent the transmission coefficients of wave modes traveling along waveguides 1 and 2 (respectively) towards the coupling interface. Finally, in Eq. (14), $\mathbb{F}$ is a $2 n \times 1$ vector expressed as

$$
\mathbb{F}=-\left[\begin{array}{cc}
\mathbf{I} & -\left(\boldsymbol{\Phi}_{\mathrm{q}}^{\mathrm{ref}}\right)_{1}^{-1}\left(\boldsymbol{\Phi}_{\mathrm{q}}^{\mathrm{ref}}\right)_{2} \\
-\left(\boldsymbol{\Phi}_{\mathrm{F}}^{\mathrm{ref}}\right)_{2}^{-1}\left(\boldsymbol{\Phi}_{\mathrm{F}}^{\mathrm{ref}}\right)_{1} & \mathbf{I}
\end{array}\right]^{-1}\left[\begin{array}{c}
\mathbf{0} \\
\left(\boldsymbol{\Phi}_{\mathrm{F}}^{\mathrm{ref}}\right)_{2}^{-1} \mathbf{F}_{e x}
\end{array}\right]
$$

The vector $\mathbb{F}$ is to be understood as a vector of generalized excitation. It can be partitioned as

$$
\mathbb{F}=\left[\begin{array}{l}
\mathbb{F}_{1} \\
\mathbb{F}_{2}
\end{array}\right]
$$

where $\mathbb{F}_{i}$ is a $n \times 1$ vector whose components denote generalized excitations for the wave modes of waveguides $i$.

Considering Eqs. (14), (17), (19) yields the following matrix formulation:

$$
\left[\begin{array}{cc|cc}
\mathbf{I} & -\mathbb{C}_{1}^{\star} \boldsymbol{\mu}^{N_{1}} & \mathbf{0} & \mathbf{0} \\
-\mathbb{C}_{11} \boldsymbol{\mu}^{N_{1}} & \mathbf{I} & \mathbf{0} & -\mathbb{C}_{12} \boldsymbol{\mu}^{N_{2}} \\
\hline-\mathbb{C}_{21} \boldsymbol{\mu}^{N_{1}} & \mathbf{0} & \mathbf{I} & -\mathbb{C}_{22} \boldsymbol{\mu}^{N_{2}} \\
\mathbf{0} & \mathbf{0} & -\mathbb{C}_{2}^{\star} \boldsymbol{\mu}^{N_{2}} & \mathbf{I}
\end{array}\right]\left[\begin{array}{c}
\mathbf{Q}_{1}^{\text {ref } \star} \\
\mathbf{Q}_{1}^{\text {ref }} \\
\hline \mathbf{Q}_{2}^{\text {ref }} \\
\mathbf{Q}_{2}^{\text {ref } \star}
\end{array}\right]=\left[\begin{array}{c}
\mathbf{0} \\
\mathbb{F}_{1} \\
\hline \mathbb{F}_{2} \\
\mathbf{0}
\end{array}\right]
$$

Solving this matrix formulation yields the wave amplitudes $\left\{Q_{1}^{\text {ref }}, Q_{1}^{\text {ref }}, Q_{1}^{\text {ref }}, Q_{1}^{\text {ref } \star}\right\}$ to be expressed. The calculation of the displacements and internal forces /moments along the waveguides - i.e. over any substructure boundary $k_{i}(i=1,2)$ - follows from the consideration of the governing equations (4) and the wave mode expansion (8). The feature of the formulation (20) is that the matrix appearing in the left hand side term is likely to be well conditioned. This is explained as the matrix involves identity submatrices on its diagonal, while the fact of right multiplying matrices of the form $\mathbb{C}$ by $\boldsymbol{\mu}^{N}$ (with $\|\boldsymbol{\mu}\|_{2}<1$ ) results in a filtering effect for spurious high order modes (additional discussions can be found in ref. [1]).

\section{Acoustic radiation}

The strategy for computing the acoustic radiation of stiffened or non-stiffened rectangular plates, using the WFE method, is proposed in this section. In the present study, those plates are supposed to be surrounded by an infinite rigid baffle while radiating in an acoustic fluid (air). Also, the plates are supposed to be excited by a point force (see previous section) while subjected to arbitrary boundary conditions of the form (6). The vibroacoustic system involving a radiating rectangular plate is depicted in Figure 3. The fluid is supposed to be inviscid and light, in the sense that its loading on the plates is neglected. 


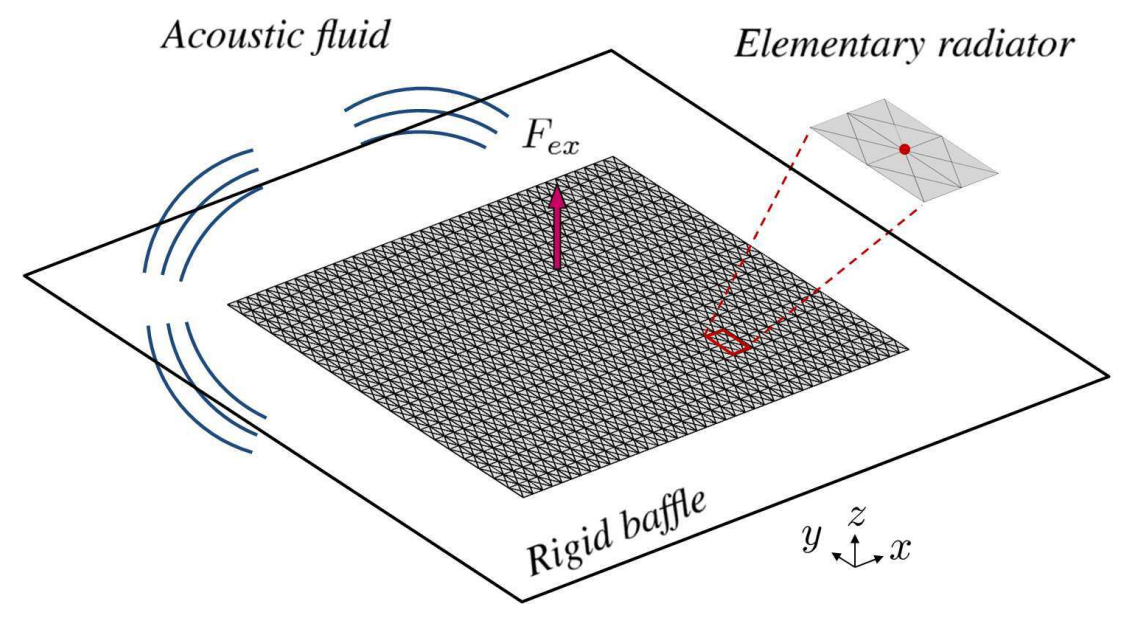

Figure 3: Illustration of a rectangular plate surrounded by a rigid baffle and radiating in a light acoustic fluid.

Within the WFE framework, the dynamic behavior of a stiffened or non-stiffened rectangular plate vibrating in vacuo is to be expressed from the strategy depicted in the previous section. Then the resulting normal velocity field of the plate is used for describing its acoustic radiation. For this kind of problem, a relevant approach is to compute the radiating power or, equivalently, the radiation efficiency. For this task, the method of elementary radiators can be used [2]. This suggests to "discretize" the plate into elementary surfaces of same area $S_{\text {radiator }}$ and constant normal velocities, and to compute the radiation efficiency as

$$
\sigma=\frac{\dot{\mathbf{q}}_{n}^{H} \mathbf{R} \dot{\mathbf{q}}_{n}}{\rho_{0} c_{0} S_{\text {plate }}<\left(\dot{\mathbf{q}}_{n}\right)^{2}>}
$$

where $\dot{\mathbf{q}}_{n}$ is the vector of normal velocities of the elementary radiators, expressed as $\dot{\mathbf{q}}_{n}=i \omega \mathbf{q}_{n}$ where $\mathbf{q}_{n}$ is the vector of normal displacements; also, $<\left(\dot{\mathbf{q}}_{n}\right)^{2}>$ is the mean quadratic velocity averaged over all the elementary radiators, defined as

$$
<\left(\dot{\mathbf{q}}_{n}\right)^{2}>=\frac{1}{2} \frac{1}{N_{\text {rad }}} \sum_{k=1}^{N_{\text {rad }}}\left|\left(\dot{q}_{n}\right)_{k}\right|^{2},
$$

where $N_{\text {rad }}$ is the total number of elementary radiators that are used for discretizing the plate, while $\left(\dot{q}_{n}\right)_{k}$ is the normal velocity of a given radiator $k$. Also, in Eq. (21), $S_{\text {plate }}$ is the area of the plate while $\mathbf{R}$ is a full square matrix whose components are

$$
R_{s t}=\frac{\omega^{2} \rho_{0} S_{\text {radiator }}^{2}}{4 \pi c_{0}} \frac{\sin \left(k_{0} r_{s t}\right)}{k_{0} r_{s t}} \quad(s \neq t) \quad, \quad R_{s s}=\frac{\omega^{2} \rho_{0} S_{\text {radiator }}^{2}}{4 \pi c_{0}},
$$

where $k_{0}=\omega / c_{0}$ is the acoustic wavenumber and $r_{s t}$ is the distance between two radiators $s$ and $t$. A typical elementary radiator is depicted in Figure 3. The normal 
velocity of each radiator is supposed to be constant and equal to the normal velocity at its mid node (cf. Figure 3). In the present framework, the WFE method can be used for describing the vector of normal displacements (and thus the vector of normal velocities) over any substructure boundary of waveguides 1 and 2 (see Section 2) as $\left(\mathbf{q}_{n}\right)_{i}=\mathcal{L}^{\prime}\left(\boldsymbol{\Phi}_{\mathbf{q}}\right)_{i} \mathbf{Q}_{i}(i=1,2)$ where $\left(\boldsymbol{\Phi}_{\mathbf{q}}\right)_{i}=\left[\left(\boldsymbol{\Phi}_{\mathbf{q}}^{\text {inc }}\right)_{i}\left(\boldsymbol{\Phi}_{\mathbf{q}}^{\text {ref }}\right)_{i}\right], \mathbf{Q}_{i}=\left[\mathbf{Q}_{i}^{\text {inc } T} \mathbf{Q}_{i}^{\text {ref } T}\right]^{T}$ is the vector of wave amplitudes and $\mathcal{L}^{\prime}$ is an incidence matrix for capturing the normal displacements at the relevant DOFs. Thus, the numerator appearing in the right hand side of Eq. (21) can be written as

$$
\begin{aligned}
\dot{\mathbf{q}}_{n}^{H} \mathbf{R} \dot{\mathbf{q}}_{n}= & \omega^{2} \sum_{i} \sum_{s \geq 1} \mathbf{Q}_{i}^{H}\left[\boldsymbol{\mu}^{\prime(s-1) H}\left(\mathbf{\Phi}_{\mathrm{q}}\right)_{i}^{H} \mathcal{L}^{\prime H} \mathbf{R}_{s s} \mathcal{L}^{\prime}\left(\mathbf{\Phi}_{\mathrm{q}}\right)_{i} \boldsymbol{\mu}^{\prime(s-1)}\right] \mathbf{Q}_{i} \\
& +2 \omega^{2} \sum_{i} \sum_{s \geq 1} \sum_{t>s} \operatorname{Re}\left\{\mathbf{Q}_{i}^{H}\left[\boldsymbol{\mu}^{\prime(s-1) H}\left(\boldsymbol{\Phi}_{\mathrm{q}}\right)_{i}^{H} \mathcal{L}^{\prime H} \mathbf{R}_{s t} \mathcal{L}^{\prime}\left(\boldsymbol{\Phi}_{\mathrm{q}}\right)_{i} \boldsymbol{\mu}^{\prime(t-1)}\right] \mathbf{Q}_{i}\right\}, \\
& +2 \omega^{2} \sum_{s \geq 1} \sum_{t \geq 1} \operatorname{Re}\left\{\mathbf{Q}_{1}^{H}\left[\boldsymbol{\mu}^{\prime(s-1) H}\left(\mathbf{\Phi}_{\mathrm{q}}\right)_{1}^{H} \mathcal{L}^{\prime H} \mathbf{R}_{s t}^{\prime} \mathcal{L}^{\prime}\left(\mathbf{\Phi}_{\mathrm{q}}\right)_{2} \boldsymbol{\mu}^{\prime(t-1)}\right] \mathbf{Q}_{2}\right\},
\end{aligned}
$$

where $\mathrm{Q}_{1}$ (resp. $\mathrm{Q}_{2}$ ) is to be understood as the vector of wave amplitudes for the radiators located at the left end of waveguide 1 (resp. right end of waveguide 2); also, $\boldsymbol{\mu}^{\prime}$ is a diagonal matrix with components $\left\{\mu_{j}^{\alpha}\right\}_{j}\left(\left\{\mu_{j}\right\}_{j}\right.$ being the wave parameters already introduced in Section 2.1), where $\alpha$ is an integer that "scales" the length $d$ of a plate substructure (see Figure 1) to the length of a radiator; finally, $\mathbf{R}_{s t}$ is a square matrix extracted from the matrix $\mathbf{R}$ (see above) and which relates the coupling between two rows of radiators $s$ and $t$ of a same waveguide $i$; $\mathbf{R}_{s t}^{\prime}$ is a square matrix extracted from the matrix $\mathbf{R}$ (see above) and which relates the coupling between two rows of radiators $s$ and $t$ belonging, respectively, to waveguides 1 and 2. Otherwise, expressing the denominator on the right hand side of Eq. (21) by means of WFE wave modes does not add any more difficulty.

Regarding Eq. (24), the feature of the WFE approach is that the matrix terms inside the square brackets do not depend on the boundary conditions of waveguides (i.e. over the left and right edges of the plate) as well as their coupling conditions. In other words, once these terms have been computed, the computation of the radiation efficiency can be achieved many times with small CPU times, e.g. to deal with parametric analysis involving several kinds of boundary and excitation conditions of the plate.

\section{Model reduction}

The idea behind the MOR strategy is to approximate the vectors of displacements $\mathbf{q}_{i}^{\left(k_{i}\right)}$ and internal forces $\mathbf{F}_{i}^{\left(k_{i}\right)}$ of each waveguide $i(i=1,2)$, over any substructure boundary $k_{i}\left(k_{i}=1, \ldots, N_{i}+1, N_{i}\right.$ being the number of substructures used for describing the waveguide $i$ ) by means of a reduced wave basis $\left\{\left(\widetilde{\boldsymbol{\Phi}}_{j}\right)_{i}\right\}_{j=1, \ldots, 2 m_{i}}$ (with a same number $m \leq n$ of incident and reflected modes). In this framework, the aim is to compute the forced response of the coupled structure using a reduced matrix 
formulation of small size $2 m$ compared to the conventional matrix formulation (whose size is $2 n$ ) obtained when the full wave bases are considered (cf Eq. (20)).

A MOR strategy that enables a reduced basis to be constructed for each waveguide $i$ in terms of the relevant wave modes - i.e. which effectively contribute for expressing the behavior of the coupled system - has been recently proposed in ref. [3]. Here we apply this strategy to the present case involving rectangular plates excited by a point force (see previous sections). In the work [3], it is shown that the relative error made for expressing the displacements and internal forces / moments of such structure involving two coupled waveguides (see previous sections) can be assessed by means of the following error bound:

$$
\begin{aligned}
\mathcal{E}_{s}=\max \{ & {\left[\left(\epsilon_{1}^{\mathbf{E}}+\epsilon_{2}^{\mathbf{E}}\right)+\frac{\left\|\mathbf{A}^{s}\right\|}{1-\left\|\mathbf{A}^{s}\right\|}\left(\epsilon_{1}^{\mathbf{A}}+\epsilon_{2}^{\mathbf{A}}\right)\right] \frac{1+\left\|\mathbf{A}^{s}\right\|}{1-\left\|\mathbf{A}^{s}\right\|}, } \\
& {\left.\left[\left(\epsilon_{1}^{\mathbf{E}^{\star}}+\epsilon_{2}^{\mathbf{E}^{\star}}\right)+\frac{\left\|\mathbf{A}^{\star s}\right\|}{1-\left\|\mathbf{A}^{\star s}\right\|}\left(\epsilon_{1}^{\mathbf{A}^{\star}}+\epsilon_{2}^{\mathbf{A}^{\star}}\right)\right] \frac{1+\left\|\mathbf{A}^{\star s}\right\|}{1-\left\|\mathbf{A}^{\star s}\right\|}\right\}, }
\end{aligned}
$$

where

$$
\begin{aligned}
& \epsilon_{1}^{\mathbf{E}}=\frac{\left\|\widetilde{\mathbf{E}}_{s}-\widetilde{\mathcal{L}} \mathbf{E}_{s}\right\|}{\left\|\mathbf{E}_{s}\right\|}, \epsilon_{2}^{\mathbf{E}}=\frac{\left\|\mathcal{L}_{\mathrm{r}} \mathbf{E}_{s}\right\|}{\left\|\mathbf{E}_{s}\right\|}, \epsilon_{1}^{\mathbf{A}}=\frac{\left\|\widetilde{\mathbf{A}^{s}} \widetilde{\mathcal{L}}-\widetilde{\mathcal{L}} \mathbf{A}^{s}\right\|}{\left\|\mathbf{A}^{s}\right\|}, \epsilon_{2}^{\mathbf{A}}=\frac{\left\|\mathcal{L}_{\mathrm{r}} \mathbf{A}^{s} \widetilde{\mathcal{L}}^{T}\right\|}{\left\|\mathbf{A}^{s}\right\|},(26) \\
& \epsilon_{1}^{\mathbf{E}^{\star}}=\frac{\left\|\widetilde{\mathbf{E}}_{s}^{\star}-\widetilde{\mathcal{L}} \mathbf{E}_{s}^{\star}\right\|}{\left\|\mathbf{E}_{s}^{\star}\right\|}, \epsilon_{2}^{\mathbf{E}^{\star}}=\frac{\left\|\mathcal{L}_{\mathrm{r}} \mathbf{E}_{s}^{\star}\right\|}{\left\|\mathbf{E}_{s}^{\star}\right\|}, \epsilon_{1}^{\mathbf{A}^{\star}}=\frac{\left\|\widetilde{\mathbf{A}^{\star s}} \widetilde{\mathcal{L}}-\widetilde{\mathcal{L}} \mathbf{A}^{\star s}\right\|}{\left\|\mathbf{A}^{\star s}\right\|}, \epsilon_{2}^{\mathbf{A}^{\star}}=\frac{\left\|\mathcal{L}_{\mathrm{r}} \mathbf{A}^{\star s} \widetilde{\mathcal{L}}^{T}\right\|}{\left\|\mathbf{A}^{\star s}\right\|}
\end{aligned}
$$

In Eq. (26), $\widetilde{\mathcal{L}}$ and $\mathcal{L}_{\mathrm{r}}$ are two incidence matrices used for the selection of the retained wave modes (i.e. the wave modes to be used in the computation of the forced response of the system) and the residual wave modes, respectively; $\mathbf{A}$ and $\mathbf{A}^{\star}$ are two $2 n \times 2 n$ matrices expressed as

$$
\mathbf{A}=\left[\begin{array}{ll}
\mathbb{C}_{1}^{\star} \boldsymbol{\mu}_{1}^{N_{1}} \mathbb{C}_{11} \boldsymbol{\mu}_{1}^{N_{1}} & \mathbb{C}_{1}^{\star} \boldsymbol{\mu}_{1}^{N_{1}} \mathbb{C}_{12} \boldsymbol{\mu}_{2}^{N_{2}} \\
\mathbb{C}_{2}^{\star} \boldsymbol{\mu}_{2}^{N_{2}} \mathbb{C}_{21} \boldsymbol{\mu}_{1}^{N_{1}} & \mathbb{C}_{2}^{\star} \boldsymbol{\mu}_{2}^{N_{2}} \mathbb{C}_{22} \boldsymbol{\mu}_{2}^{N_{2}}
\end{array}\right], \mathbf{A}^{\star}=\left[\begin{array}{cc}
\mathbb{C}_{11} \boldsymbol{\mu}_{1}^{N_{1}} \mathbb{C}_{1}^{\star} \boldsymbol{\mu}_{1}^{N_{1}} & \mathbb{C}_{12} \boldsymbol{\mu}_{2}^{N_{2}} \mathbb{C}_{2}^{\star} \boldsymbol{\mu}_{2}^{N_{2}} \\
\mathbb{C}_{21} \boldsymbol{\mu}_{1}^{N_{1}} \mathbb{C}_{1}^{\star} \boldsymbol{\mu}_{1}^{N_{1}} & \mathbb{C}_{22} \boldsymbol{\mu}_{2}^{N_{2}} \mathbb{C}_{2}^{\star} \boldsymbol{\mu}_{2}^{N_{2}}
\end{array}\right]
$$

Also, in Eq. (26), $\mathbf{E}_{s}$ and $\mathbf{E}_{s}^{\star}$ are two $2 n \times 1$ vectors expressed as

$$
\mathbf{E}_{s}=\left(\sum_{p=0}^{s-1} \mathbf{A}^{p}\right) \mathbf{B} \quad, \quad \mathbf{E}_{s}^{\star}=\left(\sum_{p=0}^{s-1} \mathbf{A}^{\star p}\right) \mathbf{B}^{\star} \quad \forall s \geq 1,
$$

where

$$
\mathbf{B}=\left[\begin{array}{c}
\mathbb{C}_{1}^{\star} \boldsymbol{\mu}_{1}^{N_{1}} \mathbb{F}_{1} \\
\mathbb{C}_{2}^{\star} \boldsymbol{\mu}_{2}^{N_{2}} \mathbb{F}_{2}
\end{array}\right] \quad, \quad \mathbf{B}^{\star}=\left[\begin{array}{l}
\mathbb{F}_{1} \\
\mathbb{F}_{2}
\end{array}\right]
$$

Also note that the vectors $\mathbf{E}_{s}$ and $\mathbf{E}_{s}^{\star}$ (Eq. (28) can be partitioned as:

$$
\mathbf{E}_{s}=\left[\begin{array}{l}
\mathbf{E}_{s 1} \\
\mathbf{E}_{s 2}
\end{array}\right] \quad, \quad \mathbf{E}_{s}^{\star}=\left[\begin{array}{l}
\mathbf{E}_{s 1}^{\star} \\
\mathbf{E}_{s 2}^{\star}
\end{array}\right]
$$


where $\mathbf{E}_{s i}$ and $\mathbf{E}_{s i}^{\star}$ are $n \times 1$ vectors associated to waveguide $i(i=1,2)$. The aforementioned matrices and vectors (Eqs. (27-29)) have been derived by expressing the vectors of wave amplitudes that result from the consideration of $s$ forward and backward passings of waves along the waveguides. When deriving the error bound $\mathcal{E}_{s}$ (Eq. (25)), it is assumed that the spectral radii of the matrices $\mathbf{A}$ and $\mathbf{A}^{\star}$ are less than one, meaning that there exists two integers $s_{0}$ and $s_{0}^{\star}$ such that $\left\|\mathbf{A}^{s}\right\|<1$ for $s \geq s_{0}$ and $\left\|\mathbf{A}^{\star s}\right\|<1$ for $s \geq s_{0}^{\star}$. In ref. [3], it has been shown that $s$ can be chosen as

$$
s=\max \left\{u \geq \max \left\{s_{0}, s_{0}^{\star}\right\}:\left\|\mathbf{A}^{u}\right\| \geq 0.1,\left\|\mathbf{A}^{\star u}\right\| \geq 0.1\right\} .
$$

Also, in Eq. (26), the tilde sign means that matrices and vectors have been formulated using a reduced wave basis (for each waveguide) instead of the full wave basis. As an additional requirement, the following assumptions $\left\|\widetilde{\mathbf{A}}^{s}\right\| \leq\left\|\mathbf{A}^{s}\right\|$ and $\left\|\widetilde{\mathbf{A}}^{\star s}\right\| \leq\left\|\mathbf{A}^{\star s}\right\|$ have to be made.

The strategy for selecting the wave modes, as proposed in ref. [3], can be stated as follows:

1. Check that $\rho(\mathbf{A})<1$ and $\rho\left(\mathbf{A}^{\star}\right)<1$ (see above);

2. Choose integer $s$ according to Eq. (31);

3. Rank the wave modes of each waveguide $i(i=1,2)$ with respect to the magnitudes of the components of vectors $\mathbf{E}_{s i}$ and $\mathbf{E}_{s i}^{\star}$;

4. Compute the error bound $\mathcal{E}_{s}$ by means of Eq. (25) at the highest frequency considered within the studied frequency band, as a function of $m$ (i.e. the first $m$ wave modes for both waveguides 1 and 2, as ranked in step 3);

5. Define the domain of validity of $\mathcal{E}_{s}$, i.e. when $\left\|\widetilde{\mathbf{A}}^{s}\right\| \leq\left\|\mathbf{A}^{s}\right\|$ and $\left\|\widetilde{\mathbf{A}}^{\star s}\right\| \leq$ $\left\|\mathbf{A}^{\star s}\right\|$ (see above);

6. Identify the minimum value of $\mathcal{E}_{s}$.

It is worth emphasizing that all these steps are to be addressed only at the highest frequency considered within the studied frequency band.

\section{Numerical experiments}

\subsection{Plate characteristics and numerical setup}

The WFE-based formulation proposed in section 3 has been applied to investigate the radiation efficiencies of baffled plates subjected to harmonic loading. For validation purposes, the characteristics of the plates are similar to those chosen by Berry et al.in 
ref. [6]. We consider rectangular baffled steel plates of dimensions $0.38 \mathrm{~m} \times 0.455 \mathrm{~m}$ $\times 0.001 \mathrm{~m}$ respectively in the $x$-, $y$ - and $z$ directions (see Figure 1), which can be either clamped or simply supported on their four edges. The chosen material properties are the following: density $\rho=7850 \mathrm{~kg} \cdot \mathrm{m}^{-3}$, Young's modulus $E=2 \times 10^{11} \mathrm{~Pa}$, Poisson's ratio $\nu=0.28$ and loss factor $\eta=0.01$. As already mentioned, the baffled plates are surrounded by an acoustic fluid and the fluid loading effect on the structure is neglected.

Each plate is subjected to a harmonic point force of unitary amplitude in the normal $z$-direction, applied at its center. As stated in section 2.2, such a force is taken into account within the WFE method by splitting the plate into two waveguides connected at the force location (the plate center in the present case). Both waveguides are modeled using a same number of substructures $N_{1}=N_{2}=10$, of length $d=0.019 \mathrm{~m}$ in the $x$-direction and width $0.455 \mathrm{~m}$. The number of DOFs contained over the left or right edges of each substructure is $n=109$ for the clamped plate, and $n=113$ for the simply supported plate. A finer discretization is also considered to improve the accuracy of the WFE-based numerical model, using substructures of half length (i.e., $d=0.0095$, that is $N_{1}=N_{2}=20$ ) having more DOFs (i.e., $n=221$ for the clamped plate, $n=225$ for the simply-supported plate).

The forced vibrations of the plates as well as their radiation efficiencies are computed over the frequency range $[10 \mathrm{~Hz} ; 3000 \mathrm{~Hz}]$ with a precision of $1 \mathrm{~Hz}$ up to 100 $\mathrm{Hz}$ and $2 \mathrm{~Hz}$ afterwards.

\subsection{Forced response computation and model reduction}

For each set of boundary conditions, the displacement $w$ in the $z$-direction at any point of the plate is computed using Eqs. (20), (3), (4). The accuracy of the WFE method to retrieve classical results of FE simulations with reduced computational time, which has been highlighted in previous papers (cf. [1] for instance), is again verified in the present cases. For example, Figure 4 compares the quadratic velocities obtained with the WFE method and the FE method at the center of the plate using identical meshes. A perfect correspondence is seen between the results of the two methods for each set of boundary conditions. The comparison of Figures 4(a) and 4(b) clearly shows that the resonance frequencies and vibration levels of the plate are strongly affected by its boundary conditions. The values of $f_{11}=52 \mathrm{~Hz}$ and $f_{11}=28 \mathrm{~Hz}$ - mentioned in ref. [6] for the resonance frequencies of the first mode, respectively for the clamped and the simply supported plate - are retrieved.

The latter results have been obtained using full te WFE formulation with wave bases, that is $n=109$ incident (or reflected) modes for the clamped plate, and $n=113$ incident / reflected modes for the simply supported plate. As stated in section 4, computational times may be even more reduced when applying the model order reduction strategy, which provides appropriate reduced wave bases. The strategy focuses on the consideration of an error bound $\mathcal{E}_{s}$ whose minimum indicates the number of wave modes to be retained [3]. The evolution of $\mathcal{E}_{s}$ (in $\%$ ) as a function of the number 


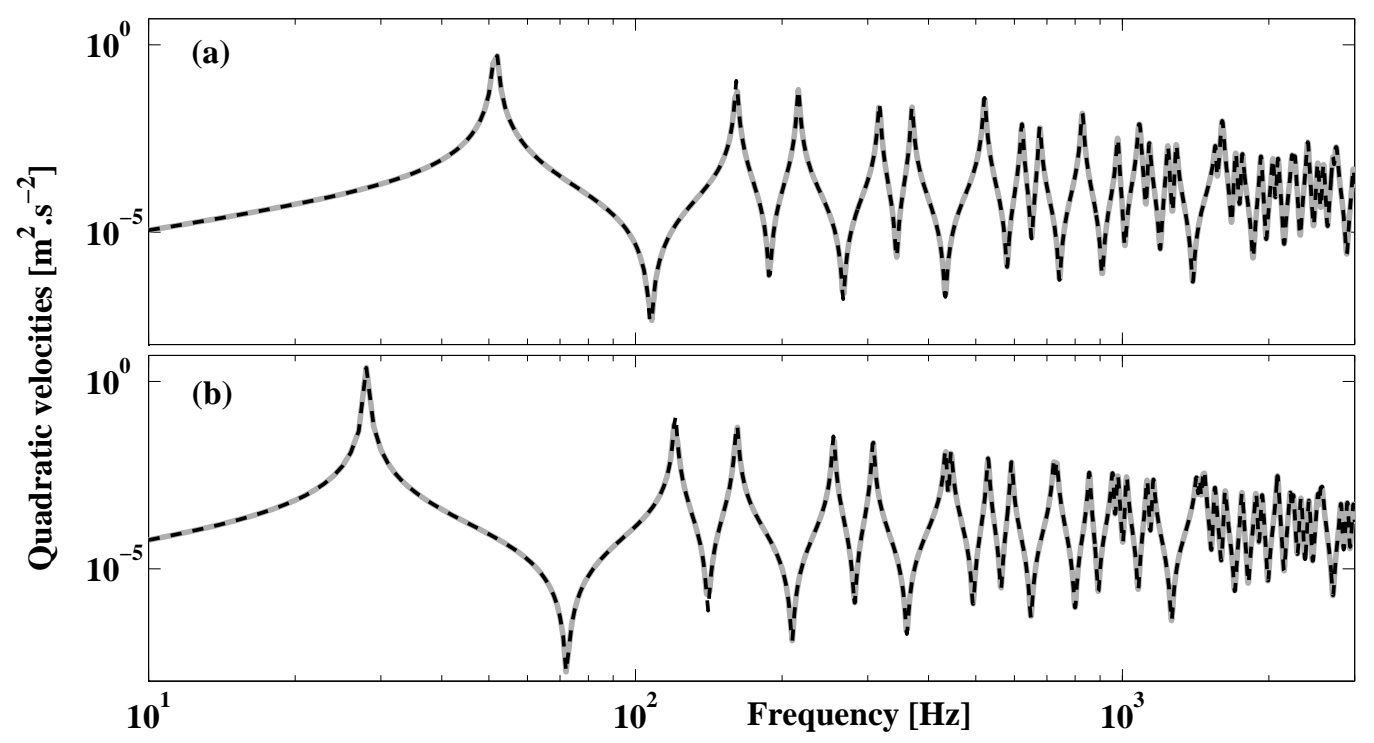

Figure 4: Center point quadratic velocity $\omega^{2}|w|^{2}$ : (- -) FE computation; (-) WFE computation; (a) clamped plate, (b) simply supported plate.

of retained wave modes is shown on Figure 5(a) considering the clamped plate with coarse mesh. The error is seen to be minimized (reaching a value of $0.16 \%$ ) when the first $m=57$ modes of the ordered full basis are retained in the reduced basis for each waveguide. It has to be noted that this minimum lies in the green shaded area, which represents the validity domain defined in section 4 (i.e., $\left\|\widetilde{\mathbf{A}}^{s}\right\| \leq\left\|\mathbf{A}^{s}\right\|$ and $\left.\left\|\widetilde{\mathbf{A}}^{\star s}\right\| \leq\left\|\mathbf{A}^{\star s}\right\|\right)$.

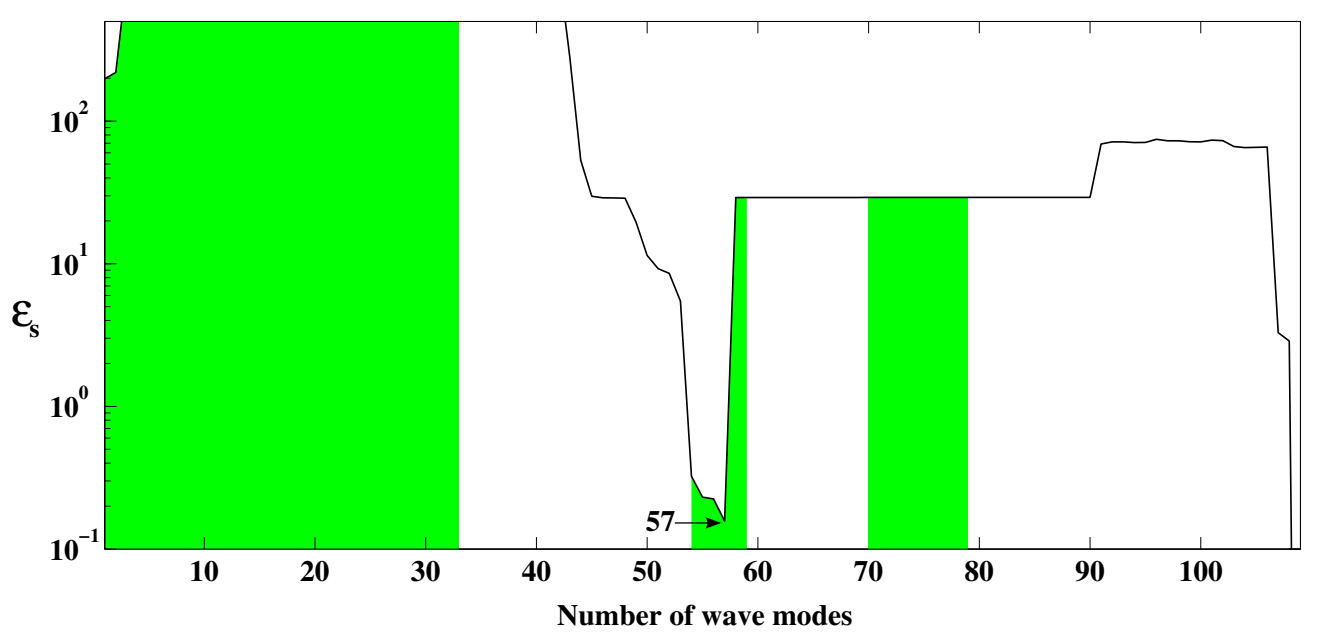

Figure 5: $\mathcal{E}_{s}(\%)$ evolution for the clamped plate with coarse mesh $(n=109)$.

This value $m=57$ represents the optimal number of wave modes required to retrieve the solution provided by the full wave basis, without overestimating the size of the reduced basis. The quadratic velocities computed using the reduced basis defined 
by the MOR algorithm are indeed exactly superimposed with the initial quadratic velocities, as displayed in Figure 6 at the plate center. An example of solution obtained using a reduced basis not provided by the MOR strategy is also shown on Figure 6; in this case, the last 10 modes have been removed from the full basis, i.e., the reduced basis contains 99 wave modes. Non negligible variations are observed at some frequencies, for instance between $2000 \mathrm{~Hz}$ and $2400 \mathrm{~Hz}$. In spite of this higher number of wave modes, the results are therefore less accurate than when using the 57 wave modes of the reduced basis provided by the MOR algorithm.

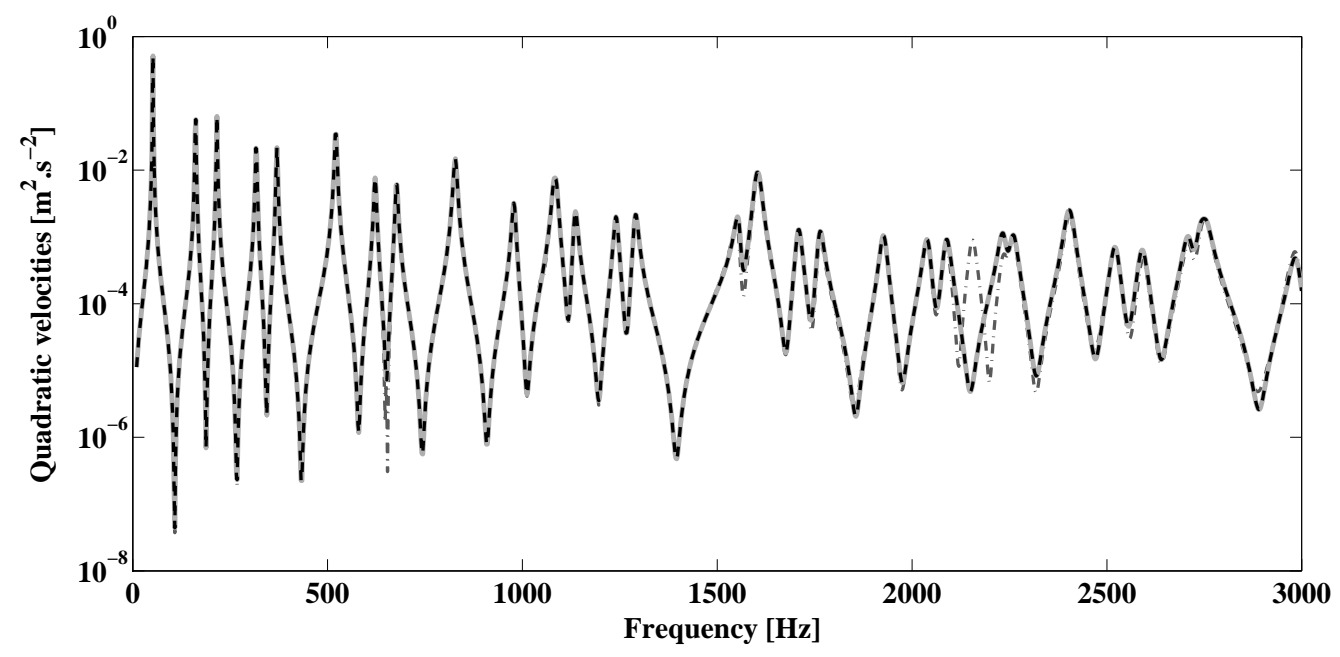

Figure 6: Center plate quadratic velocity $\omega^{2}|w|^{2}$ : (- -) full basis (109 wave modes); (-) reduced basis provided by the MOR algorithm (57 wave modes); (- . -) reduced basis obtained by removing 10 modes (99 wave modes).

\subsection{Acoustic radiation computation}

The acoustic radiation resulting from the forced vibrations of the clamped and simplysupported plates is investigated using the WFE-based formulation developed in section 3. As explained, the radiation efficiency is obtained by summing the contributions of elementary radiators uniformly distributed over the plate surface. Various numbers of radiators may be taken into account in the acoustic computations depending on the chosen substructure discretization. In the following, 140 to 560 radiators have been used, corresponding respectively to the initial and finer meshes described in section 5.1. Radiation efficiencies computations have been performed using full modal bases as well as reduced bases provided by the MOR strategy (see section 5.2). The use of the reduced bases has proved to decrease substantially the computational costs [3]. In the present case, a global time reduction of $72 \%$ has been achieved regardless of the retained number of radiators or the boundary conditions.

Figure 7 presents the radiation efficiency obtained for the plate clamped on its four edges, taking into account 560 radiators. The present result is compared to the 


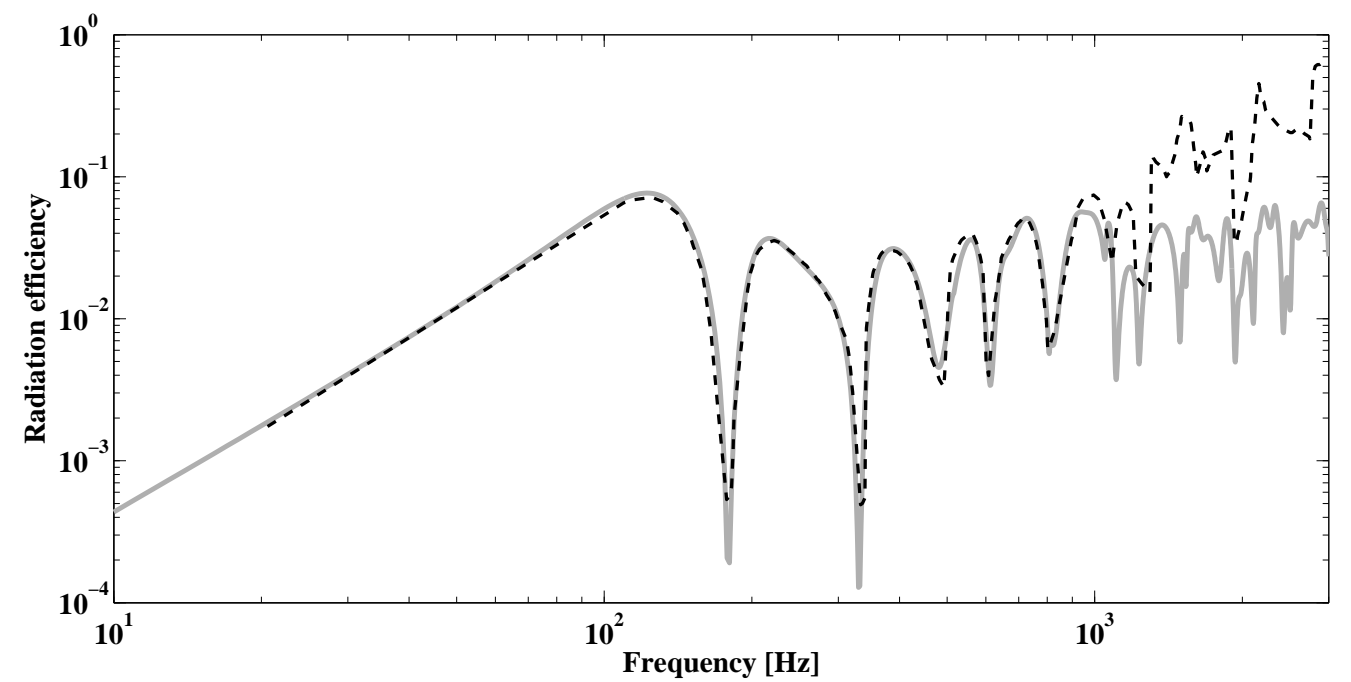

Figure 7: Radiation efficiency of the clamped plate: (- -) from Berry et al.[6]; (-) using the present approach with 560 radiators.

radiation efficiency shown by Berry et al.in [6] for the same configuration. A very good agreement is found in the low-frequency range. At upper frequencies however, the radiation efficiency computed with the present method tends to be underestimated as compared to the levels found by Berry et al. .

The same trends are observed when considering the case of the simply supported plate, shown in Figure 8. The radiation efficiency obtained with 140 radiators has

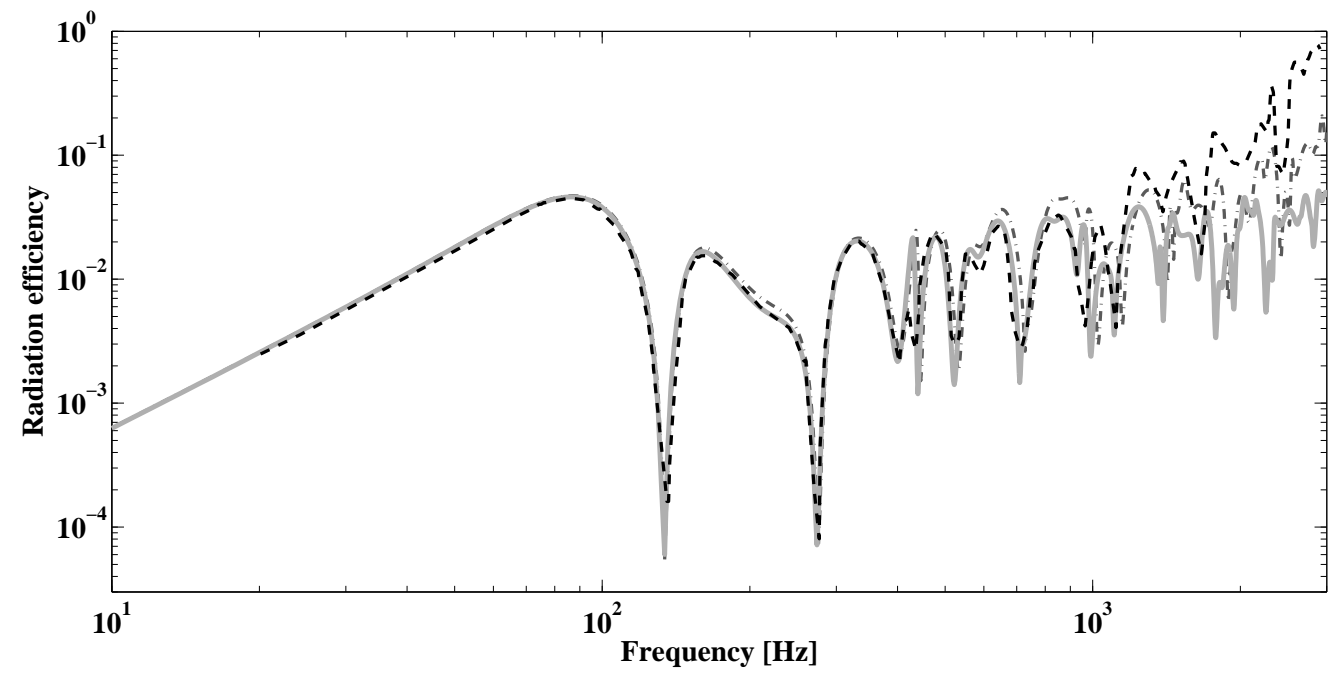

Figure 8: Radiation efficiencies of the simply supported plate: (- -) from Berry et al.[6]; (- . -) using the present approach (140 radiators); (-) using the present approach (560 radiators) . 
been displayed to investigate the influence of the number of radiators. Differences are negligible at low frequencies, while above $1 \mathrm{kHz}$ it appears that the levels may be overestimated when less information is taken into account.

Analyzing the radiation efficiencies displayed in Figures 7 and 8 also enables us to raise conclusions regarding the influence of the boundary conditions on the acoustic behavior of the plate. As expected, the radiation efficiency reaches higher levels in the case of clamped boundary conditions, although the vibration levels are slightly reduced. For example, the magnitude of the first radiation efficiency peak for the clamped plate, around $f=120 \mathrm{~Hz}$ is for instance 1.7 times higher that of the simplysupported plate (around $f=90 \mathrm{~Hz}$ ). The same approximate ratio holds for the high frequency levels.

\subsection{Acoustic radiation of stiffened plates}

The effect of stiffeners on the acoustic radiation of a plate is investigated for the case of the simply supported plate. The stiffeners are modeled as rectangular beams with cross-sectional area $16.25 \mathrm{~mm} \times 10 \mathrm{~mm}$, aligned with the $x$-direction of wave propagation. Examples of stiffened plates are shown in Figure 9. The stiffenings consist respectively of four beams spaced by $130 \mathrm{~mm}$ for the first configuration (Figure 9(a)), and ten beams spaced by $32.5 \mathrm{~mm}$ for the second one (Figure 9(b)). It is worth noting that the substructures used to compute the stiffened plates vibrations within the WFE method are therefore complex and of varying thicknesses with respect to the position in the $y$-direction. Both configurations provide the same number of DOFs on the substructure boundary, $n=109$, as for the non-stiffened plate.
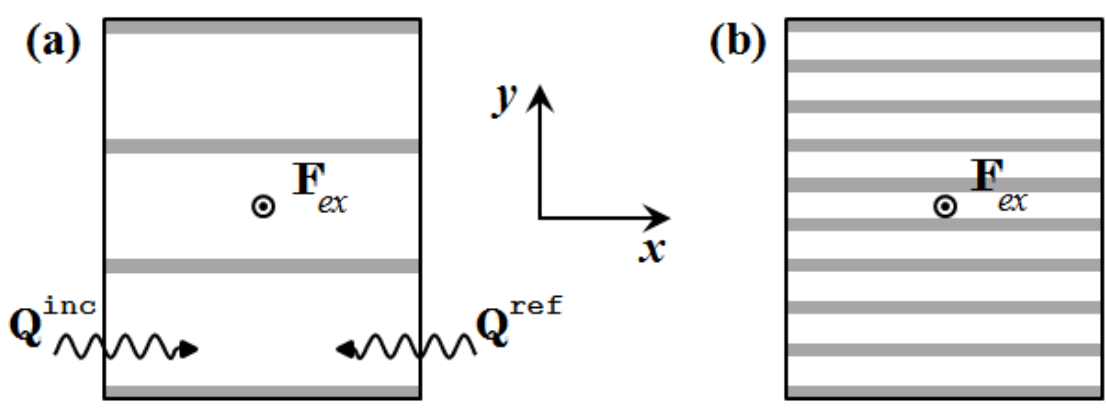

Figure 9: Stiffened plate configurations: (a) four stiffeners; (b) ten stiffeners

The influence of the stiffeners on the vibrational behavior of the plate is illustrated on Figure 10, which compares the quadratic velocities at the centers of the stiffened plates and the initial non-stiffened plate. As expected, the presence of stiffeners suppresses the vibrations at the lowest frequencies while decreasing the vibrating levels over most of the frequency range. The attenuation effect increases with the number of stiffeners. Those results are in accordance with the results presented by Nicolas 


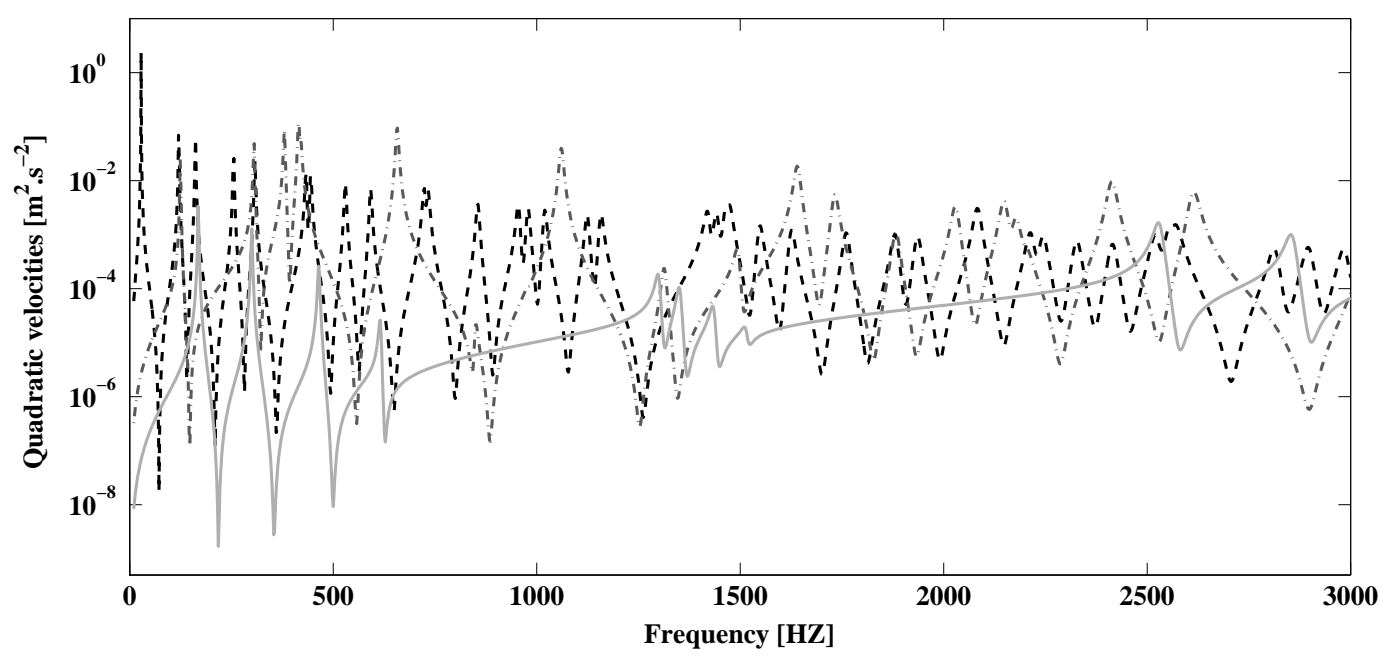

Figure 10: Center point quadratic velocities: (- -) non-stiffened plate; (- . -) plate with 4 stiffeners; (-) plate with 10 stiffeners.

and Berry in ref. [7], where a similar plate but different stiffeners sizes, shapes and positions were considered.

The influence of stiffeners on the acoustic radiation of plates is finally investigated by computing the radiation efficiencies of the above stiffened plates. The trends found in ref. [7] are again retrieved: the stiffeners are seen to considerably increase the radiation efficiency of the plate, the radiating power level being therefore reduced to a lesser extent than the vibrational level. For example, the magnitudes of the first peaks visible in Figure 11 are respectively 3.2 and 6.3 times higher for the plates with 4 and 10 stiffeners than for the non-stiffened plate, while ratios of 2.5 and 7.4 are found for the mean radiation efficiencies over the whole frequency range between the stiffened and non-stiffened plates.

\section{Conclusion}

In this paper, a formulation based on the WFE method has been developed to investigate point force driven vibrations of various rectangular plates and their radiated sound. For this purpose the plates are modeled as waveguides, that is to say periodical repetitions of a characteristic substructure in one direction of propagation. The main features of this method include:

- the computation of wave mode bases from the finite element model of the given substructure, the maximum number of modes being linked to the discretization of the substructure;

- a matrix formulation that links the DOFs at the substructure nodes (namely the normal displacements and internal forces) to the boundary conditions and external excitations; 


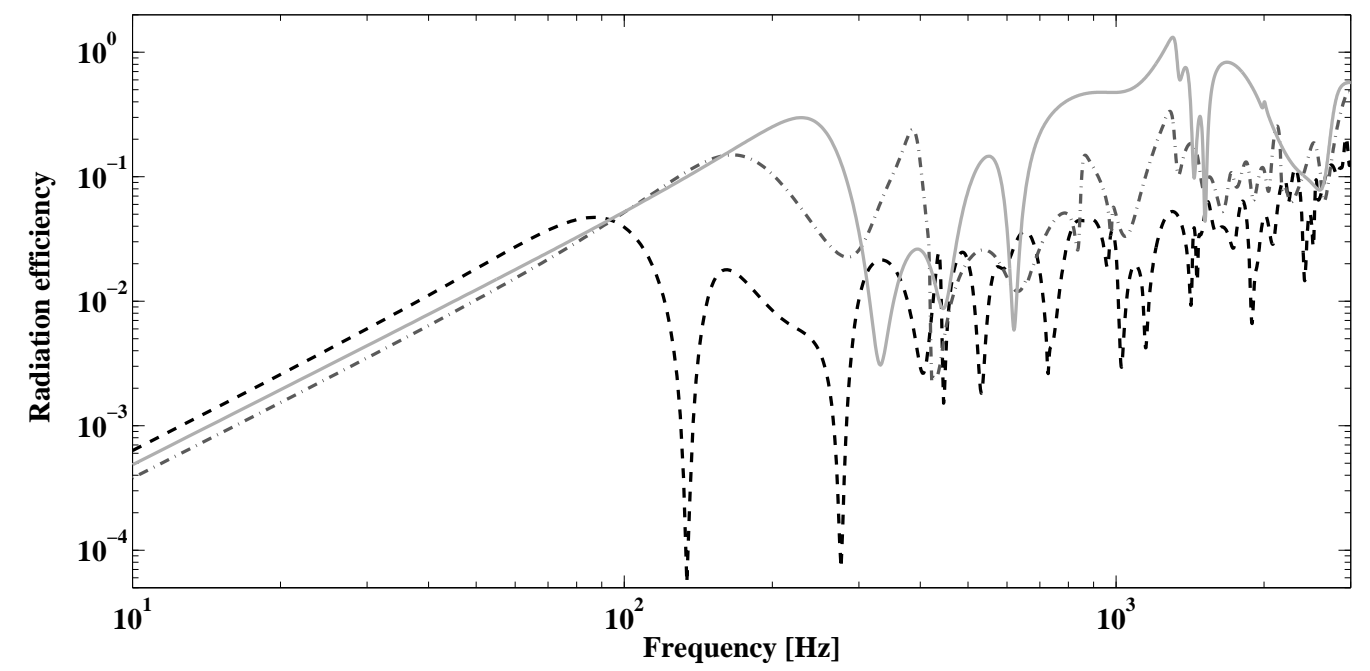

Figure 11: Radiation efficiencies: (- -) non-stiffened plate; (- . -) plate with 4 stiffeners; (-) plate with 10 stiffeners.

- the computation of the plate radiation efficiency from the normal velocities of elementary radiators uniformly distributed over the plate; those velocities are obtained from the aforementioned matrix formulation.

A model order reduction strategy has been implemented alongside. Involving matrix norm-based criteria, the algorithm provides a reduced wave mode basis which enables substantial computational time savings while obtaining similar results as when using the full basis.

The above methods have been applied to various plates of same dimensions but different boundary conditions (namely fully clamped or simply-supported plates), possibly equipped with beam stiffeners. The results in terms of vibrational levels as well as radiation efficiencies are found in good agreement with theoretical results from ref. [6] or provided by commercial software. The clamped boundary conditions is seen to slightly increase the plate resonance frequencies and radiation efficiency while reducing the vibration level, as compared with the simply-supported boundary condition. A similar trend is observed when adding stiffeners to the plate, to a greater extent. The proposed method is therefore seen to provide an efficient way of addressing vibroacoustic issues involving complex periodic structures with reduced computational costs.

\section{References}

[1] J.M. Mencik, "On the low- and mid-frequency forced response of elastic systems using wave finite elements with one-dimensional propagation", Computers and Structures, 88(11-12): 674-689, 2010. 
[2] S.J. Elliott, M.E. Johnson, "Radiation modes and the active control of sound power", Journal of the Acoustical Society of America, 94(4): 2194-2204, 1993.

[3] J.M. Mencik, "A model reduction strategy for computing the forced response of elastic waveguides using the wave finite element method", Computer Methods in Applied Mechanics and Engineering, 229-232: 68-86, 2012.

[4] D.J. Mead, "A general theory of harmonic wave propagation in linear periodic systems with multiple coupling", Journal of Sound and Vibration, 27(2): 235260, 1973.

[5] W.X. Zhong, F.W. Williams, "On the direct solution of wave propagation for repetitive structures", Journal of Sound and Vibration, 181(3): 485-501, 1995.

[6] A. Berry, J.L. Guyader, J. Nicolas, "A general formulation for the sound radiation from rectangular, baffled plates with arbitrary boundary conditions", Journal of the Acoustical Society of America, 88(6): 2792-2802, 1990.

[7] J. Nicolas, A. Berry, "Vibrations et rayonnement de plaques complexes en fluide léger et en fluide lourd", Journal de Physique IV, 2: C1-487-C1-494, 1992. 